\title{
Study on Rocky Coastline Extraction of High-Spatial-Resolution Remote Sensing Images
}

\author{
Liyan WANG, Chen HOU, Peng Li, Hui QU, Jie ZHANG \\ National Marine Data and Information Service, Tianjin 300171, China
}

\begin{abstract}
Keywords: rocky coastline extraction; remote sensing; high-spatial-resolution; threshold method Abstract. Because traditional manual visual interpretation was time and labor consuming, automatic method of rocky coastline extraction using high-spatial-resolution remote sensing data was researched. Taking WorldView-2 image as an example, interpreting marks of rocky coastline was established. By applying MNF transformation and shadow removal to the preprocessed image, a threshold segmentation model was built and threshold method was successfully utilized to extract rocky coastlines. Results show that, the proposed method performs well on rocky coastline extraction with both high precision and efficiency.
\end{abstract}

\section{Introduction}

The coastline is an adjacent transition zone between land and ocean that is the result of dynamic change processes involving natural changes and human activities ${ }^{[1,2]}$. As the fundamental geographic database of integrated coastal zone management, coastline extraction is an important part of the islands' coastal zone research ${ }^{[3]}$.

With high efficiency, wide range and dynamic characteristics, remote sensing technology has become a major means of coastline extraction ${ }^{[4]}$. Among the methods using this technique, manual visual interpretation is most commomly used. However, as it consumes so much time and labor, it is unable to meet the requirements of a wide range and rapid extraction. In addition, because the accuracy of manual visual interpretation depends on the requirements of the trace point spacing in specific projects, making the extraction results along the same area in different projects that have different precision requirements non-universal. This may cause the duplication and waste of resources.

In order to solve the problems, automatic technology on coastline extraction arises at the historic moment. However, in recent years, with high development of the remote sensing technology, a large number of high-spatial-resolution data has came into being. These data, on the one hand, brought new opportunities for the quick refinement of the coastline extraction; on the other hand, introduces a lot of noise points when using the existing methods due to the improvement of the spatial details, which seriously affect the quality of the coastline extraction. Thus, how to make full use of the high resolution remote sensing data, for providing a quick, accurate and a wide range of coastline information to the coastal zone research, has become an important problem.

According to the formation reason, the coastline can be devided into artificial coastline, rocky coastline, sandy coastline, muddy coastline, biological coastline, etc. Among them, rocky coastline extraction is of the greatest difficulties. Therefore, taking WorldView-2 images as an example, this article studies the automatic method for rocky coastline extraction using high-spatial-resolution remote sensing data. It is demonstrated that the proposed method improved the precision as well as the efficiency.

\section{Data preprocessing}

WorldView-2 data is selected to do the automatic extraction experiment of rocky coastline. Spectral feature of WorldView-2 images is shown in Tab.1. 
Tab.1 Spectral feature of worldview-2 images

\begin{tabular}{cccc}
\hline Sensor type & Band name & Central wavelength $/(\mu \mathrm{m})$ & Spatial resolution/ $(\mathrm{m})$ \\
\hline Panchromatic & PAN & ---- & 0.5 \\
Multispectral & Blue & 0.4850 & 2.0 \\
Multispectral & Green & 0.5600 & 2.0 \\
Multispectral & Red & 0.6600 & 2.0 \\
Multispectral & Near-infrared & 0.8300 & 2.0 \\
\hline
\end{tabular}

Since the original data are DN values, rdiometric calibration and atmospheric correction are performed to obtain the apparent reflectance data. In order to make full use of the panchromatic data's space information and the multispectral data's spectral information, image fusion is needed for improving the accuracy of the coastline extraction. Therefore, fusion methods, say PCA, multiply transformation, Gram-Schmidt transformation and high-pass filter, are applied and evaluation method proposed in [5] is utilized to evaluate the quality of these fusion methods. Results turn out that Gram-Schmidt transformation performs well on both spectral fidelity and spatial enhancement ability.

\section{Automatic method for rocky coastline extraction}

To extract the coastline from the remote sensing images, the first step is to extract the waterline. Then the coastline would be obtained by using tidal correction on the waterline ${ }^{[6]}$. However, the tidal correction can be ignored on the rocky coastline extraction, due to the small effects that tide shows on rocky coastlines. So, the rocky coastline extraction can be simplified into two aspects of the work. One is the establishment of remote sensing interpreting marks, and the other is the research of automatic extraction methods.

Generally, the rocky coastline locates in the obvious boundary between water and the land, and it shows up as the rock surface tonal terminator on the remote sensing image. The interpreting marks of rocky coastlines are shown in table 2.

Tab.2 Remote sensing interpreting marks of rocky coastines

\begin{tabular}{|c|c|c|c|c|c|c|}
\hline \multirow{3}{*}{ Classification } & \multicolumn{4}{|c|}{ Interpreting marks } & \multirow{3}{*}{ Location } & \multirow{3}{*}{ Images for example } \\
\hline & \multicolumn{3}{|c|}{ Direct marks } & \multirow{2}{*}{$\begin{array}{c}\text { Indirect } \\
\text { marks }\end{array}$} & & \\
\hline & Shape & Tone & Structure & & & \\
\hline Rocky coastline & Curved & $\begin{array}{l}\text { There's always a big } \\
\text { color difference on } \\
\text { the rock surface. } \\
\text { That is to say, the } \\
\text { seaside is darker and } \\
\text { the land is brighter. }\end{array}$ & $\begin{array}{l}\text { Rough } \\
\text { texture }\end{array}$ & $\begin{array}{l}\text { Always with } \\
\text { jutting cape }\end{array}$ & $\begin{array}{l}\text { Locates in } \\
\text { the } \\
\text { boundary } \\
\text { between } \\
\text { water and } \\
\text { the land. }\end{array}$ & \\
\hline
\end{tabular}

There are multiple methods for automatic coastline extraction, such as multispectral classification, threshold method ${ }^{[7]}$, edge detection ${ }^{[8]}$, region growing ${ }^{[9]}$, neural networks classification ${ }^{[10]}$, etc. Among them, the threshold method is more widely used. Because it is simple, fast and efficient, making it more suitable for a wide range of coastline extraction work. The key of this method is establishing an effective mathematical model to realize precise segmentation. Therefore, this article mainly studys on the establishment of this model and how it can be utilized to the rocky coastline extraction.

As for high-spatial-resolution remote sensing images, it's more complex to extract rocky coastlines. There are two reasons. For one thing, although there's a significant spectral difference between the dry and wet rocks on either side of the rocky coastline, it is impossible to extract the coastline directly via the threshold method. That's mainly because the different reflectivity among different rocks brings obvious tonal variation shown on the high spatial resolution images. This always leads to a large 
amount of noise points along the extracted coastlines. For another thing, as the rocky coast often comes with jutting cape and inland bay, obvious shadow appears on the remote sensing images, which makes severe influence on the interpretation accuracy and big difficulties for the coastline extraction. Therefore, it is important to reduce the noise and remove the shadows before extracting the rocky coastline.

By concentrating the main information on some previous bands, Minimum Noise Fraction (MNF) makes it possible to separate the noise from high-spatial-resolution images ${ }^{[11]}$. Then, the needed bands can be selected according to the eigenvalues. In this article, MNF transformation is used to denoise the WorldView-2 image and the first two bands are output. Results of MNF transformation are shown in figure 1.

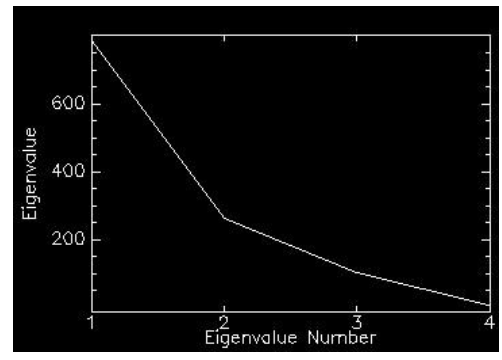

a.Eigenvalues

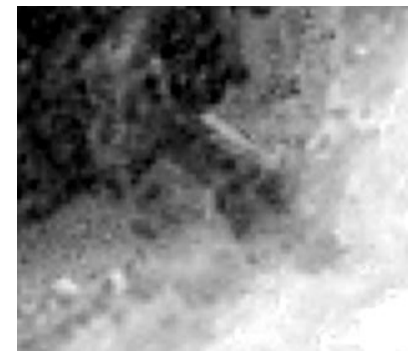

b. Band 1

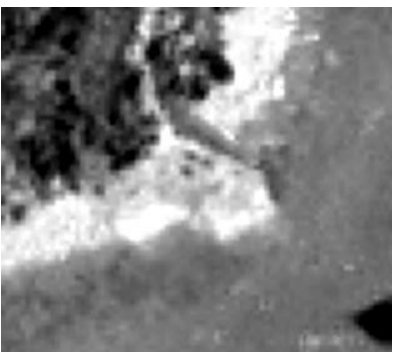

c.Band 2

Fig.1 Results of MNF transformation

It can be seen that the obtained band 2 contains rich details and shows an big intensity contrast between water and the land. The only shortage is the shadow remains. So the shadow detection and removal method proposed by Jian LIU ${ }^{[12]}$ is adopted to eliminate the impact from shadows. And then, the processed band 2 is served as the characteristic band for the threshold method. Thus, rocky coastline is finally extracted with high precision. The extracted results are shown in figure 2.
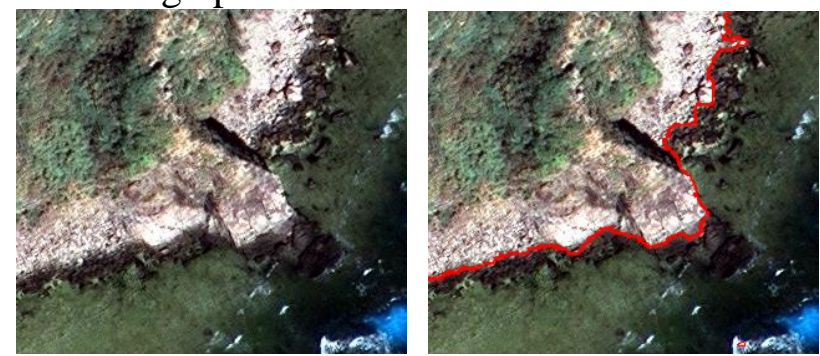

Fig.2 Results of waterline extraction

\section{Conclusions}

Taking WorldView-2 images as example, this article focuses on automatic extraction technique for rocky coastline using high-spatial-resolution remote sensing images. By testing in the experimented area, a few advantages of the proposed method is summarized:

Firstly, the extracted coastline is effective. By applying MNF transformation and shadow removal to the fusion image, a threshold segmentation model is built and the rocky coastline is extracted. The results turn out to be continuous and smooth. The noise points, which always come with the existing methods when applied to high-spatial-resolution data, are avoided.

Secondly, the extraction accuracy is much better than manual artificial interpretation. This is mainly because the proposed algorithm is based on the pixels, and the spatial resolution of the fusion image is 0.5 meters. It means that the precision of the extracted rocky coastline can reach to 0.5 meters as well. It will maximally satisfy different projects with their high accuracy requirements.

Finally, the coastline is extracted in high speed. Since the threshold segmentation method is quite simple and easy to operate, the workload is greatly reduced and the work efficiency is improved, making it more possible to obtain a wide range of coastline in a short time. 


\section{Acknowledgements}

This work was financially supported by the Global Change and Air-Sea Interaction Project (CASI-03-03-01-03).

\section{References}

[1] Dongxing Xia, Yan Duan, Sangyun Wu: Journal of Marine Sciences. Vol. 27 (2009), p. 28-33. "In Chinese"

[2] Yun Zhang, Xueming Li, Jianli Zhang, et al: Hindawi Publishing Corporation Abstract and Applied Analysis (2013).

[3] Weifu Sun, Yi Ma, Jie Zhang, et al: Bulletin of Surveying and Mapping. Vol. 3 (2011), p. 41-44. "In Chinese"

[4] Xukai Zhang, Xia Zhang, Banghui Yang, et al: Remote Sensing for Land and Resources. Vol. 25 (2013), p. 91-97. "In Chinese"

[5] Liyan Wang, Dong Zhao, Jianping Chen: Journal of Astronautics. Vol. 35 (2014), p. 938-945. "In Chinese"

[6] Jiashuang Shen, Jingsheng Zhai, Haitao Guo: Hydrographic Surveying and Charting. Vol. 29 (2009), p. 74-77. "In Chinese"

[7] J H Ryu, J S Won, K D Kung, et al: Remote Sensing of Environment. Vol. 83 (2002), p. 442-456.

[8] H Liu, K C Jwzek: International Journal of Remote Sensing. Vol. 25 (2004), p. 937-958.

[9] A J Chen, C F Chen, K S Chen: Geoscience and Remote Sensing Symposium. Vol. 3 (1995), p. 2097-2099.

[10] T W Ryan, P J Semintilli, P Yuen, et al: Photogrammetry and Remote Sensing. Vol. 57 (1991), p. 947-955.

[11] Shubin Deng: ENVI processing methods of remote sensing images. Science Press (2010). "In Chinese"

[12] Jian Liu, Zhanghua Xu, Kunyong Yu, et al: Society for Agricultural Machinery. Vol. 44 (2013), p. 238-241. "In Chinese" 